\title{
Some Examples in the Realization of Symmetry *
}

\author{
Frank Wilczek ${ }^{\mathrm{a}} \dagger$ \\ ${ }^{a}$ Institute for Advanced Study, School of Natural Science \\ Olden Lane, Princeton, NJ 08540
}

I briefly discuss three phenomena arising in recent work where the realization of symmetries in quantum mechanics is unusual. The first of these is, I believe, the very simplest realization of non-trivial confinement by a mechanism of charge-flux frustration. It arises in models having several coupled abelian Chern-Simons gauge symmetries, which closely resemble effective theories used for the quantum Hall effect. The second is symmetry obstruction by non-abelian flux, which has implications for $2+1$ dimensional supergravity. Third is the possibility of new varieties of quantum statistics: non-abelian and projective.

\section{Apology}

I don't think I have much to teach this audience about string theory as such. What I will do, that I hope is useful, is discuss a few phenomena that have come up in my recent work which are in some way connected to fundamental issues regarding the realization of symmetry in quantum mechanics. My strong impression is that the fundamental symmetry of string theory, or whatever string theory is evolving into, has yet to be fully elucidated; in trying to do so, it may be well to have the possibility of unusual realizations in mind. Also, the examples I will be considering involve in simple low-dimensional gauge field theories of a kind very similar to those often arising in world-sheet or membrane studies.

\section{Simple Models of Confinement}

A classic mechanism of confinement is abstracted from the behavior of type II superconductors [1]. These materials confine magnetic flux in tubes, so that they would induce a linear confining potential between magnetic monopoles. It has long been conjectured that an analogous mechanism could underly confinement in $3+1$ dimensional relativistic quantum field theo-

\footnotetext{
*Talk given at Strings '97, 18-21 June 1997, Amsterdam, The Netherlands. To appear in Nuclear Physics Proceedings Supplement.

${ }^{\dagger}$ Research supported in part by DOE grant DE-FG0290ER40542.
}

ries[2], and recent marvelous work has supported this idea, at least in the supersymmetric case [3]. There are, however, several significant limitations to the analogy: perhaps most important, the confinement applies to a strictly additive quantum number, and does not exhibit saturation, as one has for triality; also, the monopole is not an elementary object, and it is not in general clear that the degrees of freedom needed to construct it are available or that it is a plausible stand-in for quarks; finally, related to this, the detailed implementation tends to be complicated and indirect. Now I would like to show you a class of field theories that exhibit confinement in a very simple and transparent way through charge-flux frustration [4], and where the elementary quanta are confined and the confinement saturates. Considered as models for QCD these field theories have weaknesses of their own: they live in $2+1$ dimensions, the dynamics seems to be essentially abelian, and the confinement is only logarithmic - but at least they are different weaknesses. These theories are closely related to the effective theories used to describe hierarchical and layered states in the quantum Hall effect. From a conceptual standpoint, it is particularly interesting that they contain no massless particles in their perturbative spectrum; thus they exhibit, in a very clear-cut way, confinement arising as a global phenomenon, rather than through accumulation of infrared singularities. 
The Lagrangians of interest to us take the form

$\mathcal{L}=\frac{1}{8 \pi} \epsilon^{\alpha \beta \gamma} \mu^{l m} a_{\alpha}^{l} f_{\beta \gamma}^{m}+D_{\alpha} \phi^{r}\left(D^{\alpha} \phi^{r}\right)^{*}-V(\phi),(1)$

where the covariant derivative on the $r^{\text {th }}$ scalar field acts as $D_{\alpha} \phi^{r} \equiv \partial_{\alpha} \phi^{r}-i q_{l}^{r} a_{\alpha}^{l} \phi^{r}$ (suspending the summation convention on $r$ ), and $V$ is an effective potential whose details need not concern us. $\mu$ is a symmetric matrix. The normalization is chosen in such a way that integral entries in $\mu$ and integral charges arise naturally, for example if the theory results from breakdown of an overlying nonabelian theory.

Let us first consider the simple case when $\mu$ is a $2 \times 2$ matrix whose only non-zero entries are equal to an integer $n$ off the diagonal. Assume that just one scalar field, carrying charges $(0, q)$ with respect to the two gauge groups, condenses. The field equations of most interest to us arise from the variation of $\mathcal{L}$ with respect to the timecomponent of $a^{1}$ and with respect to the spacecomponents of $a^{2}$. Allowing for an external source carrying charge of the first type, these equations read:

$\frac{n}{2 \pi} b^{2}=\rho_{\text {ext }}^{1}$.

and

$\frac{n}{2 \pi} \vec{e}^{1}=\hat{z} \times \vec{j}^{2}$.

Let us analyze the second of these first. If we require that the condensate lives on the vacuum manifold at infinity, and is single-valued, then by a gauge transformation it can be brought into the form $\phi \rightarrow v e^{i l \theta}$, where $l$ is an integer and $v$ is magnitude of the vacuum expectation value of $\phi$. In order that the current due to the condensate fall off faster than $1 / r$ at infinity we must further require that

$l=q a_{\theta}^{2}$,

in order that the azimuthal covariant derivative vanish. Using Stokes' theorem, the second of these integrates to the flux quantization condition $2 \pi l=q \Phi^{2}$. Integrating the first equation over all space, on the other hand, gives us $Q_{\text {ext. }}^{1}=\frac{n}{2 \pi} \Phi^{2}$.

Combining these, we find the condition

$Q_{\text {ext. }}^{1}=\frac{n l}{q}$. on the external charge. If $n$ does not divide $q$, this condition will generally fail, even for integer $Q_{\text {ext. }}^{1}$. In that case, it will not be possible for the fields to fall off faster than $1 / r$ at infinity. The resulting long-range fields lead to energy diverging logarithmically with distance, proportional to the square of the fractional part of $\frac{q}{n} Q_{\text {ext. }}^{1}$. Energy of this sort will arise from the gradient energy of the condensate, or (if a Maxwell term is present in an expanded form of $\mathcal{L}$ ) the Coulomb field energy, or both. Thus charges which do not satisfy the condition [are confined. On the other hand, from the form of the condition it is clear that an appropriate multiple of a confined charge will not be confined. Thus finite-energy states will include baryon-like objects, but not the corresponding quarks.

In the more general $2 \times 2$ case, when $\mu=\left(\begin{array}{cc}m_{1} & n \\ n & m_{2}\end{array}\right)$ and the condensate has charge vector $\left(q^{1}, q^{2}\right)$, by following steps similar to those above, we find conditions

$$
\begin{aligned}
& Q_{\text {ext. }}^{1}=\frac{1}{2 \pi}\left(m_{1} \Phi^{1}+n \Phi^{2}\right)+q^{1} \lambda \\
& Q_{\text {ext. }}^{2}=\frac{1}{2 \pi}\left(n \Phi^{1}+m_{2} \Phi^{2}\right)+q^{2} \lambda
\end{aligned}
$$

and the flux quantization condition

$2 \pi l=q^{1} \Phi^{1}+q^{2} \Phi^{2}$,

for the long-ranged fields to vanish. Here $\lambda$ is a continuous parameter, representing the ability of the condensate to screen electric charge. The ratio of screening charges, of course, must follow that of the condensate. Now in the generic case, after solving the flux quantization condition, there will be two continuous parameters $\lambda$ and $\Phi^{2}$ available to satisfy the remaining two equations, and then an arbitrary charge will be screened (not confined). However if $m_{2}\left(q^{1}\right)^{2}-2 n\left(q^{1}\right)\left(q^{2}\right)+$ $m_{1}\left(q^{2}\right)^{2}=0$, then these two parameters multiply proportional coefficients. Hence there will be charges that cannot be screened, and must be confined. (Our earlier case corresponds to $m_{1}=m_{2}=q^{1}=0$.) A brief calculation reveals that the condition $Q \mu^{-1} q=l$ which must 
be satisfied by unconfined charges can be written in the transparent form

$q^{1} Q_{\text {ext. }}^{2}-q^{2} Q_{\text {ext. }}^{1}=\sqrt{-\Delta} l$,

where the determinant $\Delta \equiv m_{1} m_{2}-n^{2}$. There will be non-trivial real solutions $\left(q^{1}, q^{2}\right)$ if and only if $\Delta \leq 0$; there will be integer solutions if and only if $-\Delta$ is a perfect square.

It is amusing to see simple number-theoretic conditions emerging in confinement criteria. Similar but more intricate phenomena occur for larger numbers of species. They have not been explored systematically.

\section{Symmetry and Supersymmetry Ob- struction [5]}

A nonabelian vortex is characterized in regular gauge by a non-zero matrix vector potential $a_{\theta}$ at spatial infinity, and the holonomy $h=\mathcal{P} e^{i \int_{0}^{2 \pi} a_{\theta} d \theta}$. Here $\mathcal{P}$ denotes path ordering, and $a$ is a matrix in the adjoint representation. (More precisely, $h$ is defined only up to conjugacy, since a gauge transformation $\Lambda(r, \theta) \rightarrow$ $\Lambda_{\infty}(\theta)$ takes $h \rightarrow \Lambda_{\infty}(2 \pi) h \Lambda_{\infty}(0)^{-1}$.) We may suppose, after a non-singular gauge transformation, that $a_{\theta}$ is a constant matrix. Given a charged field $\eta$, one has its covariant derivative $D_{\mu} \eta=\partial_{\mu} \eta+i \mathrm{~T}^{a} a_{\mu}^{\mathrm{a}} \eta$. Here $\mathrm{T}^{\mathrm{a}}$ are the generators for the representation to which $\eta$ belongs, and of course the $a^{\text {a }}$ are the internal components of $a$. If one diagonalizes $\mathrm{T}^{\mathrm{a}} a_{\mu}^{\mathrm{a}}$ (assumed constant) and considers the partial wave $\eta(r, \theta)=e^{i l \theta} f(r)$, then (in the regular gauge) $l$ is quantized to be an integer, but for the $k^{\text {th }}$ eigenvalue $e_{(k)}$ and eigenvector $\eta_{(k)}$ one has the azimuthal derivative $D_{\theta} \eta_{(k)}=i\left(l+e_{(k)}\right) \eta_{(k)}$. And so the angular energy term, which has direct physical significance, is proportional to $\frac{\left(l+e_{(k)}\right)^{2}}{r^{2}}$. It behaves as if there is a fractional contribution $e_{(k)}$ to the angular momentum. Alternatively, by a singular gauge transformation (with gauge function proportional to $\theta$, and thus having a cut) one could formally remove $a_{\theta}$, at the cost of introducing boundary conditions of the form $\eta(2 \pi)=\rho(h) \eta(0)$ on the azimuthal dependence of the field $\eta$ (and thus on the wave functions of its quanta). Here of course $\rho$ is the appropriate unitary representation matrix. In this formulation the azimuthal covariant derivative is simply the ordinary spatial azimuthal derivative, but one has the condition $l_{(k)}=$ integer $+\frac{\phi_{(k)}}{2 \pi}$ on the partial waves $\eta_{(k)} \propto e^{i l \theta} f(r)$. The form of the angular energy term, of course, is the same as before, with $e_{(k)} \equiv \phi_{(k)} / 2 \pi$.

Generically states with a given value of $e_{(k)}$ (and the same spatial wave functions) will be degenerate, but states with different values of $e_{(k)}$ will not be degenerate, because they see a different effective Hamiltonian - or, alternatively, because they obey a different angular momentum quantization condition. Now suppose that the symmetry of the ground state is a non-abelian group $K$, and consider $\kappa \in K$. If $\rho$ is a faithful representation, and $h \kappa \neq \kappa h$, then $\rho(\kappa)$ will not act within the spaces of fixed $e_{(k)}$, but will connect states with different values of $e_{(k)}$. As we have seen, generically this means that $\rho(\kappa)$ will connect states of different energy. Thus the obstructed symmetry $K$ will not be realized as a symmetry of the spectrum, but will connect states with different energy. Only members of the centralizer group $\mathcal{C}(h)$ of transformations which commute with $h$ will generate spectral symmetries.

The effects of obstructed symmetry appear directly only in states carrying both flux and charge. These can be brought together in a straightforward way, as bound states, with the flux realized as a classical field. A more intrinsic form of the phenomenon occurs in ChernSimons theories, where the interaction itself naturally associates flux with charge. The question then arises whether splittings are induced for the fundamental quanta, which carry both charge and flux, and what is the mechanism. I expect a splitting does arise, for the following reason. In evaluating the self-energy of such a quantum, one must sum over space-time trajectories where its world line is self-linked or knotted. The charge from one part of the trajectory gets entangled with the flux emanating from another part, and the propagation is altered, as above. For non-dynamical sources (Wilson lines) it is known that the amplitude of a trajectory depends on its topology; in- 
deed, this fact lies at the root of the application of non-abelian Chern-Simons theories to topology. It would be very interesting to do a dynamical calculation along these lines.

In $2+1$ dimensional gravity the primary effect of a point mass is to create a conical geometry in its exterior, with a deficit angle $\delta$ proportional to the mass; $\delta=2 \pi G m$. The exterior geometry is then locally flat, but non-trivial globally. Indeed, it induces a modification in the angular momentum quantization for particles in the exterior, similar in some ways to the non-abelian vortex. A notable difference is that whereas the non-abelian vortex induced an additive change in the quantization condition, change induced by the gravitational field of a point mass is multiplicative. Indeed, from requiring that the azimuthal factor $e^{i l \theta}$ be single-valued as $\theta \rightarrow \theta+(2 \pi-\delta)$ we find $l=$ integer $/\left(1-\frac{\delta}{2 \pi}\right)$. This difference reflects the different symmetry of the sources: an additive shift in angular momentum requires an intrinsic orientation violating the discrete symmetries $\mathrm{P}$ and $\mathrm{T}$, while a multiplicative factor is even under these transformations [6].

The conical geometry presents a global obstruction to defining a spinor supercharge (see below), so that supersymmetry, even if valid for the ground state, is obstructed for massive states. This fact plays a role in some speculative ideas for using supersymmetry to zero the cosmological term, while remaining consistent with its absence in the physical spectrum [7,8]. One can reasonably expect that fermion-boson degeneracy is lifted, but a concrete calculation might be welcome. It is not altogether trivial to produce one, because the expected effect comes from a subtle infrared phenomenon, so that one needs to do some sort of non-perturbative calculation; but on the other hand the theory is non-renormalizable.

One can remove the effect of the conical geometry by a singular gauge transformation, in favor of a modified boundary condition. Here the covariant derivative should respect parallel transport, so that we should require that the effect of orbiting the apex of the cone is to rotate spinors (or vectors) through the appropriate angle, i.e. to multiply them by a phase proportional to the spin and angle. An important subtlety, which arises even in flat space, is that one must include a factor -1 for half-odd integer (fermion) fields. This factor is essentially equivalent to the -1 accompanying fermion loops in Feynman graphs, and its inclusion implements the normal spin-statistics relation. With this factor $e^{2 \pi i s}$ taken out, the appropriate condition on wave functions $\psi_{s} \propto f(r) e^{i l \theta}$ for spin $s$ quanta, in order that they transform properly, is $\psi_{s}(2 \pi-\delta)=e^{i(2 \pi-\delta) l-i \delta s} \psi_{s}(0)$ leading to

$l=\frac{1}{1-\frac{\delta}{2 \pi}}$ integer $+s \frac{\frac{\delta}{2 \pi}}{1-\frac{\delta}{2 \pi}}$.

This superelectrodynamics can be coupled to supergravity, and one can consider gravitational corrections to the 'hydrogen' spectrum. One can, in this context, consider the corrections to the effective non-relativistic Hamiltonian due to one photino exchange. The computation closely follows the classical derivation of the Breit Hamiltonian for positronium and yields the correction

$\Delta \mathcal{H}=\frac{-i}{2 m}\left[P_{i}, V(x)\right] \Sigma_{i}$,

where $m$ is the electron mass, $V$ is the Coulomb potential $\frac{e^{2}}{2 \pi} \ln r$, and the $\Sigma_{i}$ are matrices acting in the internal spin space of the electron-proton system. The form of this correction to the Hamiltonian is not altered by gravity, since $\Delta \mathcal{H}$ is a local operator on the wavefunction and gravity in $2+1$ dimensions has only global, topological effects. This is consistent with the boundary conditions mentioned above; indeed the exact form of the matrices $\Sigma_{i}$ implies that, in a conical geometry, the operator $\Delta \mathcal{H}$ becomes hermitian, in a non-trivial way. The spin matrices connect components of the wavefunction with internal spin differing by 1 , and the phase acquired by the operator $\left[P_{i}, V(x)\right]$ (or simply by $x_{i}$ after taking the commutator), which would otherwise spoil hermiticity, is exactly compensated by the different, spin-dependent, boundary conditions on these components.

The main effect of the gravitational field, however, arises already in the nominally spinindependent interaction, which dominates in the low-velocity (non-special relativistic) limit: the 
modified condition for the allowed angular momenta modifies the effective Schroedinger equation, and splits the spectrum corresponding to different spin values. Here we are concerned with the motion of the electron in the geometry and potential provided by the proton, and take $\delta=2 \pi G M$. Clearly supersymmetry is obstructed, and it is not manifest in the spectrum.

We have glossed over several technicalities that do not substantially affect this leading-order calculation, though in extending it to higher orders in $e^{2}$ and $m / M$ they would need careful attention. The most interesting, perhaps, comes from the double-conical geometry arising when one includes the gravitational fields of both particles. One then obtains a modified quantization condition on the relative angular momentum, which takes the form $l_{\text {rel. }}=\frac{\text { integer }}{1-\frac{\delta_{\max }}{2 \pi}}+\frac{1}{2 \pi} \frac{\left(\delta_{1} s_{2}+\delta_{2} s_{1}\right)}{1-\frac{\delta_{\max }}{2 \pi}}$, where $\delta_{i}, s_{i}$ are the deficit angle and spin associated with particle $i$, and $\delta_{\max }$ is the larger deficit angle. Also one should use the Kerr geometry to take into account the spin and angular momentum of the particles, but these effects are subleading, of relative order $(s$ or $l) \delta$.

\section{Nonabelian and Projective Quantum Statistics}

Exotic forms of quantum statistics, apart from the familiar examples of boson and fermion, are a logical possibility inherent in the quantum kinematics of identical particles[9. In general, the issue is how to add the amplitudes for processes described by topologically distinct classes of trajectories. The classical Lagrangian, which is designed to yield the equations of motion at its extrema, does not necessarily answer this question, and one can imagine a range of models having a single classical limit but fundamentally different quantum behavior. This occurs for QCD, in connection with the $\theta$ parameter. There it is the topology of trajectories in field space which is relevant; but the same question arises even for trajectories of identical point-like particles, and this is the case we associate with quantum statistics.

This problem takes a special form in low dimensions. In $2+1$ dimensions the topological classes of trajectories are parametrized by the braid group, and one can classify the possibilities for quantum statistics by studying the unitary or, in principle, projective - representations of that group. The one-dimensional representations of the braid group describe anyons. The quasiparticles and quasiholes of the conventional Laughlin and 'hierarchical' fractional quantum Hall states are anyons, and not simply bosons or fermions.

It is natural, in this context, to ask if nonabelian representations of the braid group also occur in physics. Their implications are quite unusual. Since their irreducible representations are multi-dimensional, nonabelian representations of quantum statistics require a degeneracy in Hilbert space for the particles at fixed positions in physical space, with adiabatic transport of the particles around one another (in the absence of any explicit interaction) giving not merely a phase factor, but generally a new state.

Remarkably, it seems that this sort of thing does occur, at least theoretically, in another class of candidate quantum Hall states 10. These are states that in a sense combine the characteristic Laughlin ordering with a form of BCS pairing. The prototype state of this type is the so-called 'Pfaffian' state, which occurs at filling fraction $\nu=1 / 2$ (note the odd denominator, which cannot occur for a conventional hierarchical state). In this system, $2 n$ quasiholes fill out an irreducible $2^{n-1}$ dimensional representation of the braid group. Notice that the non-trivial structure begins only when there are at least four of these particles.

I think it is fair to say that the mathematical and physical understanding of these non-abelian statistics is still at a primitive stage. The dimension of the representations can be inferred from convincing but indirect and heuristic arguments using conformal field theory or by a rather painful explicit construction. (As I write this, a more tractable version of the conformal field theory is being developed[11.) The braiding properties have been worked out explicitly for four particles. There are strong indications that when more particles are involved they naturally fall into a spinor representation in Hilbert space. This property is eerily reminiscent of D-particle quantum mechanics, where the statistics become promoted to a 
continuous group.

Experimentally, neither the unusual abelian nor the nonabelian statistics has yet been tested directly. Indeed it is only quite recently that the fractional charge of Hall effect quasiparticles was convincingly observed, in shot noise experiments [12]. The situation is far from hopeless, however.

In higher dimensions, the braid group is no longer relevant - one has room to untangle the braids - and one is dealing simply with the symmetric group. This group of course supports nonabelian representations, leading to the concept of parastatistics. There is another possibility, however, that is (in contrast to parastatistics) remarkably unique and extends smoothly to arbitrary numbers of particles. This is the possibility of projective statistics. As I shall now discuss, it also leads to spinor representations closely related to what arises in the Pfaffian state.

It is a general feature of quantum mechanics that symmetries of the observables are implemented by unitary transformations of Hilbert space. However, this implementation need not respect the group multiplication law, when there is a group of symmetries. Since the observables are not affected by a phase redefinition of the states, all that is required is $U\left(g_{1}\right) U\left(g_{2}\right)=$ $\eta\left(g_{1}, g_{2}\right) U\left(g_{1} g_{2}\right)$, in an obvious notation; the $\eta \mathrm{s}$ are complex numbers of modulus unity.

The projective representations of the symmetric group are related to ordinary representations of an extended group I shall write as $\tilde{S}_{N}$, where $N$ is the number of identical particles. $\tilde{S}_{N}$ plays, in this regard, the same role for the symmetric group $S_{N}$ as $S U(2)$ plays for the rotation group $S O(3) . \quad \tilde{S}_{N}$ is most easily defined using generators and relations. One introduces elements $T_{i}, 1 \leq i \leq N-1$, and $z$ satisfying $T_{i}^{2}=1$, $\left(T_{i} T_{i+1}\right)^{3}=1$, and $T_{i} T_{j}=z T_{j} T_{i}$ for $|i-j| \geq 2 . z$ is a central element with $z^{2}=1$. One should think of $T_{i}$ as implementing the transposition $(i, i+1)$. If $z=1$ these relations define the symmetric group itself; so the irreducible representations of $\tilde{S}_{N}$ with $z=1$ simply reproduce the ordinary irreducible representations of $S_{N}$. The other possibility is $z=-1$. This corresponds to projective representations proper. From the lin- ear representation of $\tilde{S}_{N}$ one obtains a projective representation of $S_{N}$ by choosing, for every permutation in $S_{N}$, some definite way of obtaining it as a product of the transpositions $T_{i}$. The choice is unique up to a sign ambiguity, which cannot be removed. A very curious aspect of the properly projective representations is that the order of interchanges, even between distinct and possibly distant particles, affects the phase of the wave function; this is a direct consequence of the third defining relation for $\tilde{S}_{N}$.

The projective representations of $S_{N}$, or equivalently the linear representations of $\tilde{S}_{N}$, were determined by Schur in a series of remarkable papers, even before the advent of quantum mechanics 13, 14. The properly projective representations, like the ordinary representations, are associated with Young tableaux. There is the new feature, however, that the length of the rows must be strictly decreasing. Thus there is a projective analog of the bosonic tableaux (one row of length $N$ ) but not of the fermionic tableaux ( $N$ rows of length one).

The simplest, quasi-bosonic projective representation is already quite richly structured, and naturally involves spinors in $N$ dimensions. One can realize it, although not quite irreducibly, using a set of $N+1$ gamma matrices $\Gamma_{i}$, and defining $T_{i}=\frac{1}{\sqrt{2}}\left(\Gamma_{i}-\Gamma_{i+1}\right)$. By a change of basis we can make do with $N$ matrices, defining $T_{i}=\sqrt{\frac{i-1}{2 i}} \Gamma_{i-1}-\sqrt{\frac{i+1}{2 i}} \Gamma_{i}$. In this form, the representation is irreducible. Its size grows exponentially with the number of particles, as $2^{[N / 2]-1}$, just matching the corresponding degeneracy of quasiholes in the Pfaffian state. This sort of growth can be interpreted as the emergence of a new two-valued degree of freedom with each added pair of particles. Such a picture can be made more explicit by representing the $\Gamma$ matrices in terms of fermion creation and annihilation operators, as $\Gamma_{2 i-1}=a_{i}+a_{i}^{*}, \Gamma_{2 i}=i\left(a_{i}-a_{i}^{*}\right)$.

In closing I would like to add one more general observation on this topic. In the generic quantum theory, the observables are independent of an overall phase rotation, and this opens the possibility of projective realizations of symmetry. In gauge invariant systems the observables are inde- 
pendent of local gauge transformations; in principle, this opens up the possibility of much wider classes of 'improper' realizations of quantum symmetry.

\section{REFERENCES}

1. See for example J.R. Schrieffer, Theory of Superconductivity, (Benjamin-Cummings, 1964; Third Printing, Revised, 1983, Reading, Mass., 1983); P. de Gennes, Superconductivity of Metals and Alloys, (Benjamin-Cummings, Reading, Mass., 1966).

2. S. Mandelstam, Phys. Rep. 23, 245 (1976); G. 'tHooft, Nucl. Phys.B190, 455 (1981).

3. Reviewed, for example, in M. Shifman, "Nonpertubative Dynamics In Supersymmetric Gauge Theories", Lectures given at International School of Physics, 'Enrico Fermi', Course 80: Selected Topics in Nonperturbative QCD, Varenna, Italy, 27 Jun - 7 Jul 1995, and at Summer School in High-energy Physics and Cosmology, Trieste, Italy, 10 Jun - 26 Jul 1996. hep-th/9704114.

4. L. Cornalba and F. Wilczek, Phys. Rev. Lett. 254679 (1997); M. de Wild Propitius, "Confinement in Partially Broken Abelian ChernSimons Theories," to be published in Phys. Lett. B, hep-th/9704063.

5. This section closely follows L. Cornalba and F. Wilczek, "Mass Splittings From Symmetry Obstruction," to be published in Phys. Lett. B, hep-th/9706014.

6. For further information on $2+1$ Gravity, see R. Jackiw, Planar Gravity, in Diverse Topics in Theoretical and Mathematical Physics, (World Scientific, Singapore, 1995); F. Mansouri, Phys. Lett. B372 (1996); F. Mansouri, Phys. Lett. B397 81 (1997).

7. E. Witten, Int. J. Mod. Phys. A10, 1247 (1995); E. Witten, Mod. Phys. Lett. A10, 2153 (1995).

8. K. Becker, M. Becker, and A. Strominger, Phys. Rev. D51 6603 (1995).

9. For overviews of these matters, including reprints of many of the original papers, see F. Wilczek, Fractional Statistics and Anyon Superconductivity (World Scientific, 1990), and
M. Stone, The Quantum Hall Effect (World Scientific, 1992).

10. G. Moore and N. Read, Nucl. Phys. B360 362 (1991); C. Nayak and F. Wilczek, Nucl. Phys. B479 529 (1996).

11. E. Fradkin, C. Nayak, A. Tsevlik and F. Wilczek, paper in preparation.

12. R. de-Picciotto, M. Reznikov, M. Heiblum, V. Umansky, G. Bunin and D. Mahalu, Nature, 389, 162 (1997).

13. I. Schur, J. Reine Angew. Math. 13285 (1907); I. Schur, J. Reine Angew. Math. 139 155 (1911).

14. For a brief introduction, see M. Hamermesh, Group Theory and Its Application to Physical Problems, Chapter 12, (Dover Publications, Inc., New York, 1962); For a modern mathematical treatment, see P. N. Hoffman and J. F. Humphreys, Projective Representations of the Symmetric Groups: Q-Functions and Shifted Tableaux, (Oxford University Press, New York, 1992). 